\title{
Lachen verbindet, ist ansteckend, schenkt Glücksmomente
}

\author{
Ursula Steiner-König \\ Dr. med., FMH-Delegierte ins Zentralkomitee Tag der Kranken
}

Als im Zentralkomitee des Tag der Kranken die Idee aufkam, Humor als Thema für den nächsten Tag der Kranken als Motto zu wählen, waren alle begeistert: einmal etwas Positives, nichts Krankheitszentriertes! Auch etwas, was für restlos alle Menschen zugänglich ist! Das sagt sich so leicht, aber: Wie zaubere ich ein Lächeln auf das Gesicht eines Schwerkranken?

Es kann nicht darum gehen, Witze zu erzählen, denn ich weiss nicht, was mein Gegenüber als Witz verstehen und ertragen würde. Es geht auch nicht darum, ein homerisches Gelächter auszulösen, noch um sarkastische oder zynische Bemerkungen. Diese sind meist entwertend, verletzend oder lenken von einer Begegnung von Person zu Person ab. Vergessen wir nie: Humor und Lachen sind nicht identisch!

«To analyze humour is a task as delicate as analyzing the composition of a perfume with its multiple ingredients, some of which are never consciously perceived while others, when sniffed in isolation, would make one wince.» So nachzulesen in den Kommentaren zum englischen humour in der Encyclopædia Britannica. Lachen sei andererseits eine biologische Erscheinung, einzig und allein geschaffen, damit sich Spannung auflösen könne. Dazu passt, was der deutsche Schriftsteller Wilhelm Raabe gesagt haben soll: Humor sei der Schwimmgürtel auf dem Strom des Lebens.

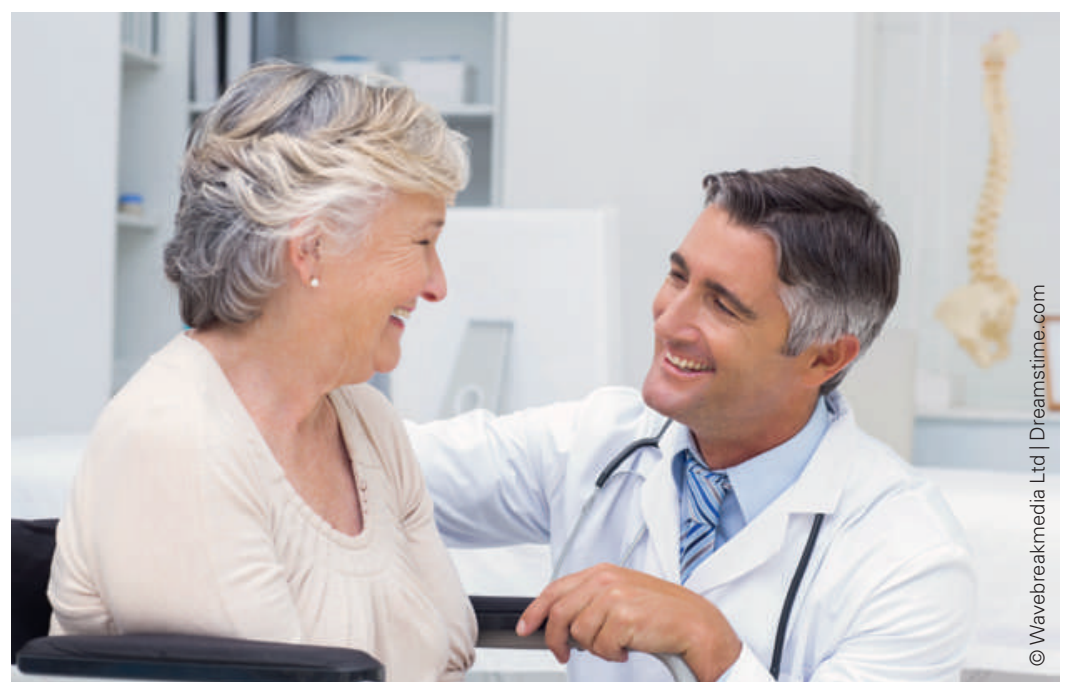

Lachen entspannt und lockert die Gedanken.
Was man erreichen möchte: Glücksmomente schenken, indem es gelingt, so auf die leidende Person einzugehen, dass ein erleichtertes Lächeln bei ihr aufscheint. Dabei geht es darum, eine neue (vielleicht sogar schräge) Perspektive zu vermitteln, die sanft die Gedanken auflockert. Vergleichbar der Reaktion eines Kleinkinds, das angelacht wird (man weiss, dass das Lachen in einer Hirnregion ausgelöst wird, die deutlich älter ist als das Sprachzentrum). Lächeln als Zeichen des Angenommen-Seins, des Verstanden-Werdens, einer echten Begegnung.

Die Humorforschung zeigt aber auch auf, dass Regionen der Grosshirnrinde einbezogen sind, die erst spät in der Entwicklung reifen. Dies bedeutet, dass höhere kortikale Funktionen keine unwesentliche Rolle spielen (siehe z.B. Barbara Wild).

\section{Der salutogenetische Aspekt}

Es lässt sich nicht feststellen, wann der salutogenetische Aspekt von Lachen nicht nur erkannt, sondern auch beschrieben und als Praxis sogar systematisiert wurde. Hingegen finden sich seit etwa zwanzig Jahren zunehmend Publikationen über Untersuchungen, was Humor, Heiterkeit, Lächeln oder Lachen alles bewirken kann. Zielgruppen sind etwa Schmerzpatienten, Demente, schwerkranke Kinder auf Palliativ-Stationen, aber auch in der Sozialarbeit wird der Einsatz von Humor beforscht. Eigentlich kann seine entspannende, aggressionsabbauende und stressmindernde Wirkung überall da, wo es um Mensch-zu-Mensch-Beziehungen geht, zum Einsatz kommen. Sogar der Satiriker Andreas Thiel meint: «Humor ist die höchste Form von Erkenntnis», und präzisiert: «Humor ist die Erkenntnis, dass nichts so ernst ist, wie es scheint.»

Die Humortherapie-Spezialisten gründeten 1998 in Basel, anlässlich des 3. Basler Humorkongresses, den Verein HumorCare für den deutschsprachigen Raum. 2001 wurde er in unabhängige Ländergesellschaften aufgeteilt. Inzwischen machen in der Schweiz auch die Stiftungen Humor \& Gesundheit sowie Theodora mit unterschiedlichem Schwerpunkt auf sich aufmerksam. 
Interessant ist zudem, dass - erstaunlicherweise - zu Beginn der neunziger Jahre gleichfalls in Indien ein Arzt, Madan Kartaria, auf die gesundheitsfördernde Wirkung von Lachen aufmerksam wurde. In seinem Land entwickelten sich die Lachgruppen, welche in der Öffentlichkeit abgehalten wurden, als kostensparende Präventions-Angebote! Er machte die Erfahrung, dass besser als Witze erzählen das Nutzen der physiologischen Mechanismen des Lachens, also eines Kontext-losen Lachens, zum Erfolg führte.

Physiologisch ist bekannt, dass beim Lachen nicht nur die Gesichtsmuskulatur, sondern auch Rumpfmuskeln und Zwerchfell aktiviert werden. Herzfrequenz und Sauerstoffsättigung nehmen beim Lachen zu. Es werden weniger Stresshormone, jedoch vermehrt Endorphine (Glückshormone) ausgeschüttet. Gemäss einer Untersuchung entsprechen 20 Sekunden Lachen etwa der körperlichen Leistung von 3 Minuten schnellem Rudern (das ist allerdings nicht das Ziel eines Einsatzes von Lachen bei Kranken).

Am Krankenbett braucht es viel Einfühlungsvermögen, damit eine Steigerung des Wohlbefindens erreicht werden kann. Alles Spöttische, Entwertende, Verletzende wäre daher fehl am Platz. Im Rahmen der Persönlichkeitspsychologie untersucht etwa das Psychologische Institut der Universität Zürich seit 1980 Persönlichkeitsunterschiede in der Perzeption und Rezeption von Humor. Es zeigte sich beispielsweise, dass Extrovertierte mehr lachen und leicht zum Lachen zu bringen sind. Personen mit heiterem Charakter berichten über weniger körperliche Symptome und erweisen sich als resilienter gegen Stress.

\section{Lächeln als Zeichen des Angenommen- Seins, des Verstanden-Werdens, einer echten Begegnung.}

\section{Das psychologische Potential des Humors}

Es lassen sich (gemäss Salameh) drei Dimensionen der psychotherapeutischen Wirkung des Humors unterscheiden:

Emotional: hemmungslösend, verdrängte Affekte reaktivierend, einen unmittelbaren und spontanen Austausch menschlicher Gefühle ermöglichend.

Kognitiv: kreative Potentiale anregend, für neuartige Zusammenhänge sensibilisierend, hilft, rigide Verhaltensmuster durch flexiblere zu ersetzen.

Kommunikativ: Humor wirkt erfrischend, trägt zu einer freundlich-konstruktiven Beziehung bei, fördert ein Klima der Offenheit und Gleichwertigkeit.

Eine noch nicht publizierte Studie des Psychologischen Instituts der Universität Zürich bei dementen Men- schen zeigt beispielsweise, dass von Herzen kommendes Lächeln differentiell von weniger spontanen Formen von Lächeln oder Lachen unterschieden und herausfiltriert werden kann (ein Bericht dazu aus CURAVIVA findet sich auf der Website Tag der Kranken).

\section{Der sogenannte medizinische Humor}

Grobe und/oder sarkastische Witze funktionieren als Entlastung vom eigenen schweren Betroffen-Sein und Erleben in Zusammenhang mit schweren Schicksalsschlägen von Patienten und Patientinnen. Derartige Äusserungen/Sprüche im Kollegenkreis wären in der Begegnung mit Kranken völlig fehl am Platz. In der SÄZ hat Samia Hurst im Herbst 2015 auf die «dunkeln Seiten des medizinischen Humors» hingewiesen. Er dürfe sein, man bräuchte sich seiner nicht zu schämen. Aber wir müssen uns des Besonderen bewusst sein und damit umgehen können.

In der Comedy-Szene lassen sich vermutlich vergleichbare Abläufe registrieren. Auch dort ist Lachen nicht unschuldig. Der Schweizer Satiriker Andreas Thiel gibt $\mathrm{zu}$, es falle ihm leichter, etwas Lustiges über Folter, Krieg oder Terror zu schreiben als über Humor - da wären wir wieder beim Anfang!

Es gibt keine evidenzbasierte "Guideline of Best Practice» für tastendes Beschnuppern des kranken, leidenden Gegenübers, um hoffentlich die richtige Düftemischung herauszufinden. Wir bewegen uns auf der bio-psycho-sozialen Achse. Der neue Ansatz, wie ihn B. Kissling im Schweizerischen Medizin-Forum unter «Qualitative Qualität» skizziert, ist daher sehr zu begrüssen! Es brauche eine wissenschaftliche Zusammenarbeit mit den Geistes- und Sozialwissenschaften. Das kommt nicht nur den Kranken, sondern auch den Behandelnden zugute. Daher: ein Hauch von Poesie, die richtige Duftmischung und vielleicht dazu noch eine Melodie ...

Literatur zum Thema

- Michael Titze (Hrsg). Kleinbasel und der «Humor in der Therapie», HCDVerlag Tuttlingen, 2013: mehrere Artikel.

- Samia Hurst. Wenn man nicht mal mehr lachen kann .. Schweiz Ärztezeitung. 2015;96(40):1466.

- Daniele Muscionico. Interview mit Andreas Thiel. NZZ vom 6. Nov. 2015: http://www.nzz.ch/feuilleton/humor-diehoechste-form-von-erkenntnis-1.18641881

- Barbara Wild (Hrsg.). Humor in Psychiatrie und Psychotherapie. Schattauer-Verlag Stuttgart, 2012 u.a. Zitat von Wilhelm Raabe.

- Salameh W. zitiert in Peter Hain: Humor als therapeutische und soziale Kompetenz. www.humor.ch/phain/phkompetenz.html

- Madan Kataria: www.humor.ch oder www.laughteryoga.com

- Bruno Kissling. Qualitative Qualität. Schweiz Med Forum. 2015;(50/51):1172-3.

- Wikipedia: Therapeutischer Humor.

- Leuenberger Beat. Fahnden nach dem Duchenne-Lächeln. Curaviva 7-8/13.

- Website Tag der Kranken: www.tagderkranken.ch 